\title{
The regulated secretory pathway in neuroendocrine cells
}

\author{
Rafael Vazquez-Martinez ${ }^{1,2 *}$ and Stéphane Gasman ${ }^{3 *}$ \\ Department of Cell Biology, Physiology and Immunology, Instituto Maimónides de Investigaciones Biomédicas de Córdoba (IMIBIC), Reina Sofia University \\ Hospital, University of Córdoba, Córdoba, Spain \\ ${ }^{2}$ CIBER Fisiopatología de la Obesidad y Nutrición (CIBERobn), Córdoba, Spain \\ ${ }^{3}$ Centre National de la Recherche Scientifique (CNRS UPR 3212), Institut des Neurosciences Cellulaires et Intégratives (INCI), Université de Strasbourg, Strasbourg, \\ France \\ *Correspondence: bc2vamar@uco.es; gasman@inci-cnrs.unistra.fr
}

Edited and reviewed by:

Hubert Vaudry, University of Rouen, France

Keywords: secretion, neuroendocrine cells, membrane trafficking, large dense core vesicles, regulated exocytosis, endocytosis, super-resolution microscopy

The regulated secretory pathway shared by excitable cells, including neurons and neuroendocrine cells is an intricate process that comprises multiple, tightly regulated steps. After their synthesis in the endoplasmic reticulum, hormones and neuropeptides have to be sorted and packed into large dense core vesicles (also named secretory granules) in the Golgi apparatus. Granules are transported toward the plasma membrane in a cytoskeleton-dependent manner and mature into competent organelles for secretagogueinduced exocytosis. Granules are then tethered to the plasma membrane, docked, and primed, before finally releasing their contents after fusing with the plasma membrane. To ensure that neurotransmission and neuroendocrine secretion operate correctly, all these steps must be tightly regulated and coordinated both spatially and temporally. Currently, when the field of intracellular trafficking has been honored by the 2013 Nobel Prize in Physiology or Medicine (awarded to James Rothman, Randy Schekman, and Thomas Südhof for their pioneering works on vesicular transport), this issue of Frontiers in Neuroendocrine Science is aimed to providing an up-to-date overview of the cellular and molecular mechanisms governing the regulated secretory pathway in neuroendocrine cells. Reviews presented here are widely covering this topic, from the architecture of the organelle involved in secretory cargo processing and sorting, the biogenesis of secretory granules, their specific transport toward the plasma membrane to the late steps of exocytosis, and the secretory granule membrane recapture.

Early stages of the secretory pathway have been discussed by two groups. Emma Martinez-Alonso and colleagues discuss the Golgi complex architecture, as well as the regulatory proteins that govern extra- and intra-Golgi transport, and the still controversial, but not mutually exclusive, theoretical models proposed to explain cargo progression through the Golgi stack (1). The group of Richard Mains discusses the specific roles of cytosolic adaptor proteins such as AP-1A, PACS-1, and GGAs in the assembly and maturation of secretory granules (2).

Closer to the cell surface, several groups discuss the molecular mechanisms regulating the late stage of exocytosis. The group of Frédéric Meunier reviews recent insights of the role of the cortical acto-myosin network (3), whereas the role of different tethering and priming factors such as CAPS, Munc13, and Doc2 proteins is described by the groups of Ury Ashery and Tom Martin $(4,5)$. Paanteha Moghadam and Meyer Jackson review how various synaptotagmins regulate fusion pore kinetics and control the mode of release (6). Lipids have emerged as key players of the regulated exocytosis and the group of Nicolas Vitale presents an overview on the diverse roles that lipids play in defining exocytotic sites, both by affecting membrane topology and by regulating secretory vesicle priming and fusion (7).

Finally, exocytosis cannot exist without a compensatory membrane intake process (i.e., endocytosis), which allows recycling of granule components and maintains organelle integrity. The groups of Stéphane Gasman and Alla Rynditch discuss the mechanisms that coordinate clathrin-mediated compensatory endocytosis with exocytosis, highlighting the specific role of the intersectin family of scaffold proteins in exocytosis and endocytosis $(8,9)$. The group of Ana-Maria Cardenas reviews the pleiotropic role of the mechano-GTPase dynamin-2, on intracellular membrane fission and fusion events, vesicle traffic, and cytoskeleton dynamics, as well as the impact of dynamin-2 mutations on the correct functioning of the secretory pathway (10).

On a more physiological point of view, Maria-Luisa DuránPasten and Tatiana Fiordelisio present an example of how pituitary gonadotrophs receive and transduce extracellular signals to promote luteinizing ( $\mathrm{LH}$ ) and follicle-stimulating (FSH) secretion, highlighting the tremendous plasticity of the system for adapting to different physiological demands (11). Wei-Jye Lin and Stephen Salton report that single nucleotide polymorphisms in genes encoding secreted proteins are associated with neuropsychiatric or endocrine/metabolic disorders (12). Finally, Jennifer Fitch-Tewfik and Robert Flaumenhaft demonstrate how the regulated secretory pathway is similar in mast cells compared to neuroendocrine cells from the adrenal gland (13), and Burton Dickey's group describes the regulatory mechanism of mucin secretion in a non-neuroendocrine cell model (14).

On a more technical point of view, recent improvements in detection technologies, especially in optical microscopy, continually push the limits of sensitivity and resolution. The groups of Colin Rickman and Ute Becherer discuss how advances over the last decade in fluorescence microscopy provided spatial and temporal details on the subcellular organization of the molecular machinery governing the regulated secretory pathway $(15,16)$, whereas the group of Rory Duncan describes how the combination of new imaging approaches with super-resolution microscopy and novel calcium indicators is appropriate for accurate study of 
voltage-gated calcium channel locations, interactions, dynamics, and composition in living cells (17).

Collectively, this compilation of reviews intends to illustrate the recent progress made to understand the complex regulation of the granule secretory pathways in neuroendocrine cells. We are grateful to all the authors who have contributed to this Research Topic and to the dedicated reviewers who helped us reaching the highest quality standards.

\section{REFERENCES}

1. Martinez-Alonso E, Tomas M, Martinez-Menarguez JA. Morpho-functional architecture of the Golgi complex of neuroendocrine cells. Front Endocrinol (2013) 4:41. doi:10.3389/fendo.2013.00041

2. Bonnemaison ML, Eipper BA, Mains RE. Role of adaptor proteins in secretory granule biogenesis and maturation. Front Endocrinol (2013) 4:101. doi:10.3389/ fendo.2013.00101

3. Papadopulos A, Tomatis VM, Kasula R, Meunier FA. The cortical actomyosin network: from diffusion barrier to functional gateway in the transport of neurosecretory vesicles to the plasma membrane. Front Endocrinol (2013) 4:153. doi:10.3389/fendo.2013.00153

4. Friedrich R, Gottfried I, Ashery U. Munc13-1 translocates to the plasma membrane in a Doc2B- and calcium-dependent manner. Front Endocrinol (2013) 4:119. doi:10.3389/fendo.2013.00119

5. James DJ, Martin TF. CAPS and Munc13: CATCHRs that SNARE vesicles. Front Endocrinol (2013) 4:187. doi:10.3389/fendo.2013.00187

6. Moghadam PK, Jackson MB. The functional significance of synaptotagmin diversity in neuroendocrine secretion. Front Endocrinol (2013) 4:124. doi:10. 3389/fendo.2013.00124

7. Ammar MR, Kassas N, Chasserot-Golaz S, Bader MF, Vitale N. Lipids in regulated exocytosis: what are they doing? Front Endocrinol (2013) 4:125. doi:10.3389/fendo.2013.00125

8. Gubar O, Morderer D, Tsyba L, Croise P, Houy S, Ory S, et al. Intersectin: the crossroad between vesicle exocytosis and endocytosis. Front Endocrinol (2013) 4:109. doi:10.3389/fendo.2013.00109

9. Houy S, Croise P, Gubar O, Chasserot-Golaz S, Tryoen-Toth P, Bailly Y, et al. Exocytosis and endocytosis in neuroendocrine cells: inseparable membranes! Front Endocrinol (2013) 4:135. doi:10.3389/fendo.2013.00135
10. Gonzalez-Jamett AM, Momboisse F, Haro-Acuna V, Bevilacqua JA, Caviedes P, Cardenas AM. Dynamin-2 Function and dysfunction along the secretory pathway. Front Endocrinol (2013) 4:126. doi:10.3389/fendo.2013.00126

11. Duran-Pasten ML, Fiordelisio T. GnRH-induced Ca signaling patterns and gonadotropin secretion in pituitary gonadotrophs. Functional adaptations to both ordinary and extraordinary physiological demands. Front Endocrinol (2013) 4:127. doi:10.3389/fendo.2013.00127

12. Lin WJ, Salton SR. The regulated secretory pathway and human disease: insights from gene variants and single nucleotide polymorphisms. Front Endocrinol (2013) 4:96. doi:10.3389/fendo.2013.00096

13. Fitch-Tewfik JL, Flaumenhaft R. Platelet granule exocytosis: a comparison with chromaffin cells. Front Endocrinol (2013) 4:77. doi:10.3389/fendo.2013.00077

14. Adler KB, Tuvim MJ, Dickey BF. Regulated mucin secretion from airway epithelial cells. Front Endocrinol (2013) 4:129. doi:10.3389/fendo.2013.00129

15. Bost A, Pasche M, Schirra C, Becherer U. Super-resolution microscopy in studying neuroendocrine cell function. Front Neurosci (2013) 7:222. doi:10.3389/ fnins.2013.00222

16. Graczyk A, Rickman C. Exocytosis through the lens. Front Endocrinol (2013) 4:147. doi:10.3389/fendo.2013.00147

17. Hiersemenzel K, Brown ER, Duncan RR. Imaging large cohorts of single ion channels and their activity. Front Endocrinol (2013) 4:114. doi:10.3389/fendo. 2013.00114

Conflict of Interest Statement: The authors declare that the research was conducted in the absence of any commercial or financial relationships that could be construed as a potential conflict of interest.

Received: 10 March 2014; accepted: 24 March 2014; published online: 08 April 2014. Citation: Vazquez-Martinez R and Gasman S (2014) The regulated secretory pathway in neuroendocrine cells. Front. Endocrinol. 5:48. doi: 10.3389/fendo.2014.00048

This article was submitted to Neuroendocrine Science, a section of the journal Frontiers in Endocrinology.

Copyright (c) 2014 Vazquez-Martinez and Gasman. This is an open-access article distributed under the terms of the Creative Commons Attribution License (CC BY). The use, distribution or reproduction in other forums is permitted, provided the original author(s) or licensor are credited and that the original publication in this journal is cited, in accordance with accepted academic practice. No use, distribution or reproduction is permitted which does not comply with these terms. 